\title{
FINITE STRIP MODELING FOR OPTIMAL DESIGN OF REINFORCED CONCRETE FOLDED PLATE STRUCTURES *
}

\author{
$U D C 624.012 .45: 519.6=111$
}

\author{
Dragan D. Milašinovićn ${ }^{\#}$, Danica Goleš \\ University of Novi Sad, Faculty of Civil Engineering Subotica, Serbia \\ \#ddmil@gf.uns.ac.rs
}

\begin{abstract}
The optimal design of reinforced concrete folded plate structures under multiple loading conditions is presented. The design variables include geometrical quantities, like the thickness, dimensions of the structural members (plates), and topological parameters, which define the locations and the connectivity of such elements. The structural analysis required during the design process is performed by using the classical finite strip method and complex harmonic coupled finite strip method for the solution of the corresponding geometric nonlinear design problem. Some applications to the optimal design of prismatic folded plates show the effectiveness of the proposed procedures.
\end{abstract}

Key words: finite strip method, folded plate structures, reinforced concrete.

\section{INTRODUCTION}

Design, construction and management of every engineering system usually involve several technological and managerial decisions aimed to minimize the required effort or maximize the desired benefit. In structural design, the importance of this optimization process is emphasized when the scheme of the carrying mechanism follows the shape of the structure itself, becoming in this way a direct expression of its functional requirements. Clearly, the complexity of the design choices involved in this phase depends on the topology of the considered structural system.

There is a great deal of structures for which both the geometry and the material properties can be considered constants along a main direction, straight or curved, while, generally, only the loading distribution may vary (e.g. thin-walled beams, cylindrical and prismatic shell roofs [1] and box-girder bridges [2, 3]). In many cases, the performance of

Received October 24, 2012

* Acknowledgement: The work reported in this paper is a part of the investigation within the research projects: ON174027 "Computational Mechanics in Structural Engineering" and TR 36017 "Utilization of by-products and recycled waste materials in concrete composites in the scope of sustainable construction development in Serbia: investigation and environmental assessment of possible applications", supported by the Ministry for Science and Technology, Republic of Serbia. This support is gratefully acknowledged. 
these structures is also improved by means of proper longitudinal prestressing systems. For these structures, the design process should lead to define the optimal morphology of the transversal cross-section, which means its geometry, size, shape and topology, as well as the layout of the prestressing system, described by the prestressing forces and the cables profile.

In such context, the attention of this paper is focused on the optimal design of reinforced concrete structures composed by folded plates and subjected to multiple loading conditions, Fig. 1a). A proper modeling of these structures can be found within the framework of the finite strip method (FSM). As well known, this method is based on the formulation of a special class of finite elements that are as long as the structure and interconnected along the nodal lines that constitute the sides of the strips themselves. As for many other classical modeling techniques, eventual prestressing systems can also be properly modeled by means of a set of loads equivalent to the prestressing actions taking both instantaneous and time dependent losses into account.

The FSM was originally developed by Cheung [4] and was widely used by other authors for understanding and predicting the behavior of cold-formed steel members and for bridge decks. The well known uncoupled FSM formulation, represents a semi-analytical finite element process. As far as linear analysis is concerned, it takes advantage of the orthogonality properties of harmonic functions in the stiffness matrix formulation.

However in the case of the geometric nonlinear analysis, and the geometric stiffness matrix calculation, the integral expressions contain the product of trigonometric functions with higher-order exponents, and here the orthogonality characteristics are no longer valid. All harmonics are coupled, and the stiffness matrix order and bandwidth are proportional to the number of harmonics used. This kind of FSM analysis is named the HCFSM [5, 6].

The contemporary alternatives to perform the stability analysis of plate structures are generalized beam theory (GBT) [7], finite element method (FEM) and the FSM. The only difference between FEM and FSM consist of the different discretization of the structures. The FEM uses a mesh that uses discretization of the structure transversally and longitudinally, while the FSM needs only transversal discretization, using currently either harmonic or spline functions in the longitudinal direction of the structure.

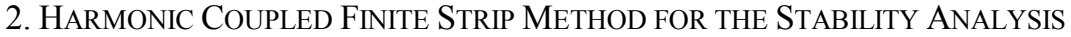

\subsection{Introduction}

If a structure undergoes large deformation, the second order terms regarding the strains cannot be ignored. The strain-displacement relations, within the context of a Green-Lagrange strain tensor, represent a sum of linear and non-linear part. Analysis of plates in the postbuckling range is generally performed on the basis of von Karman equations or by employing an energy approach. Only approximate solutions can be obtained, taking into account the in-plane (membrane) and out-of-plane (bending) boundary conditions.

The nonlinear strain-displacement relations in the finite strip can be predicted by the combination of the plane elasticity and the Kirchhoff plate theory. Using this assumption in the Green-Lagrange strain tensor (1) for in-plane nonlinear strains gives Green-Lagrange HCFSM formulation. Also that, neglecting lower-order terms in a manner consistent with the usual von Karman assumptions gives HCFSM von Karman formulation.

$$
\varepsilon_{i j}=1 / 2\left(u_{i, j}+u_{j, i}+u_{k, i} u_{k, j}\right)
$$


The essential feature of geometric nonlinearity is that equilibrium equations must be written with respect to the deformed geometry - which is not known in advance.
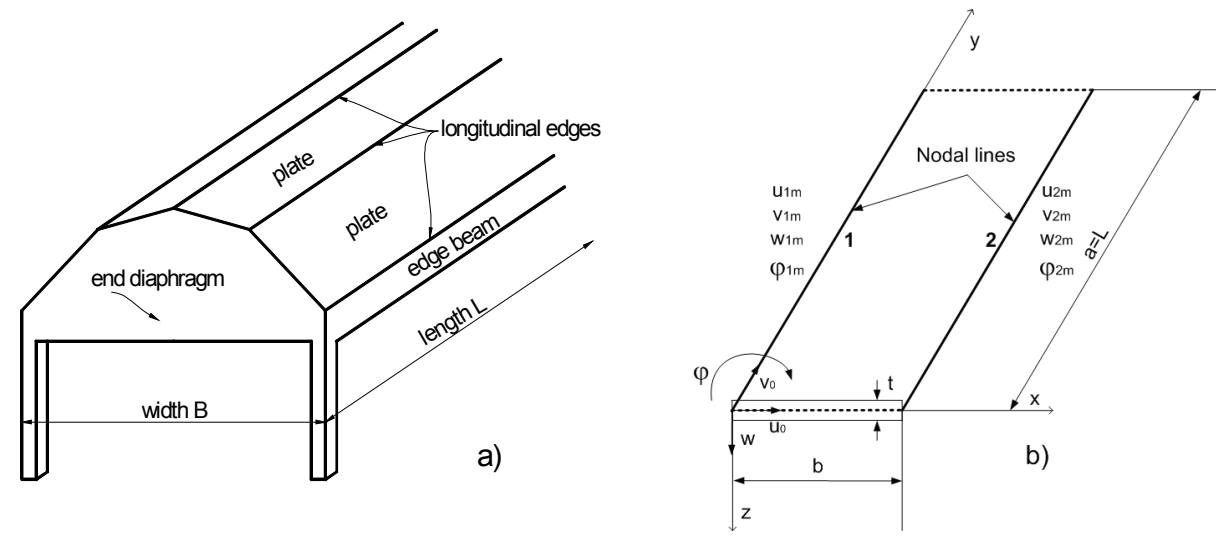

Fig. 1. a) Elements of prismatic folded plate structure, b) Simply supported flat shell strip

In the FSM, which combines elements of the classical Ritz method and the FEM, the general form of the displacement function can be written as a product of polynomials and trigonometric functions:

$$
f=\sum_{m=1}^{r} Y_{m}(y) \sum_{k=1}^{c} \mathbf{N}_{k}(x) \mathbf{q}_{k m}
$$

where $Y_{m}(y)$ are functions from the Ritz method and $\mathbf{N}_{k}(x)$ are interpolation functions from the FEM. We define the local Degrees Of Freedom (DOFs) as the displacements and rotation of a nodal line $(\mathrm{DOFs}=4)$, as shown in Fig. 1b). The DOFs are also called generalized coordinates.

The following approximate functions are used for the simply supported flat shell strip with edges restrained against in-plane movement:

$$
\begin{aligned}
& u_{0}=\sum_{m=1}^{r} Y_{u m}^{u} \mathbf{N}_{u}^{u} \mathbf{q}_{u m}^{u}=\sum_{m=1}^{r} Y_{u m}^{u}\left[\begin{array}{ll}
1-\frac{x}{b} & \frac{x}{b}
\end{array}\right] \mathbf{q}_{u m}^{u}, \\
& v_{0}=\sum_{m=1}^{r} \frac{a}{m \pi} Y_{u m}^{v} \mathbf{N}_{u}^{v} \mathbf{q}_{u m}^{v}=\sum_{m=1}^{r} \frac{a}{m \pi} Y_{u m}^{v}\left[\begin{array}{ll}
1-\frac{x}{b} & \frac{x}{b}
\end{array}\right] \mathbf{q}_{u m}^{v}, \\
& v_{0}=\sum_{m=1}^{r} \frac{a}{m \pi} Y_{u m}^{v} \mathbf{N}_{u}^{v} \mathbf{q}_{u m}^{v}=\sum_{m=1}^{r} \frac{a}{m \pi} Y_{u m}^{v}\left[1-\frac{x}{b} \quad \frac{x}{b}\right] \mathbf{q}_{u m}^{v}, \\
& w=\sum_{m=1}^{r} Y_{w m} \mathbf{N}_{w} \mathbf{q}_{w m}=\sum_{m=1}^{r} Y_{w m}\left[\begin{array}{llll}
N_{1} & N_{2} & N_{3} & N_{4}
\end{array}\right] \mathbf{q}_{w m}, \\
& N_{1}(x)=1-3\left(\frac{x}{b}\right)^{2}+2\left(\frac{x}{b}\right)^{3}, \quad N_{2}(x)=b\left[\frac{x}{b}-2\left(\frac{x}{b}\right)^{2}+\left(\frac{x}{b}\right)^{3}\right] \text {, }
\end{aligned}
$$




$$
\begin{gathered}
N_{3}(x)=3\left(\frac{x}{b}\right)^{2}-2\left(\frac{x}{b}\right)^{3}, N_{4}(x)=b\left[-\left(\frac{x}{b}\right)^{2}+\left(\frac{x}{b}\right)^{3}\right], \\
\mathbf{q}_{w m}=\left[\begin{array}{c}
w_{1 m} \\
\varphi_{1 m} \\
w_{2 m} \\
\varphi_{2 m}
\end{array}\right], \quad \mathbf{q}_{u m}^{u}=\left[\begin{array}{l}
u_{1 m} \\
u_{2 m}
\end{array}\right], \quad \mathbf{q}_{u m}^{v}=\left[\begin{array}{c}
v_{1 m} \\
v_{2 m}
\end{array}\right], \\
Y_{u m}^{u}(y)=\sin \left(m \pi \frac{y}{a}\right)=Y_{w m}, \quad Y_{u m}^{v}=Y_{u, y m}^{u}=\left(\frac{m \pi}{a}\right) \cos \left(\frac{m \pi y}{a}\right), m=1,2 \ldots
\end{gathered}
$$

\subsection{Total potential energy}

As a preliminary to tracing the equilibrium paths, it is necessary to determine the total potential energy of the structure as a function of the global DOFs. The steps in the computation are detailed discussed in [5].

The total potential energy of a strip is designated $\Pi$ and is expressed with respect to the local DOFs by the HCFSM.

$$
\begin{aligned}
& \Pi=U+W=\left(U_{m}+U_{b}\right)+W=\left(1 / 2 \int_{A} \mathbf{q}_{u}^{T} \mathbf{B}_{u 1}^{T} \mathbf{D}_{11} \mathbf{B}_{u 1} \mathbf{q}_{u} d A+1 / 2 \int_{A} \mathbf{q}_{w}^{T} \mathbf{B}_{w 3}^{T} \mathbf{D}_{22} \mathbf{B}_{w 3} \mathbf{q}_{w} d A\right)+ \\
& +\left[\begin{array}{l}
1 / 8 \int_{A} \mathbf{q}_{w}^{T} \mathbf{B}_{w 2}^{T} \mathbf{W}^{T} \mathbf{B}_{w 1}^{T} \mathbf{D}_{11} \mathbf{B}_{w 1} \mathbf{W} \mathbf{B}_{w 2} \mathbf{q}_{w} d A+1 / 4 \int_{A} \mathbf{q}_{w}^{T} \mathbf{B}_{w 2}^{T} \mathbf{W}^{T} \mathbf{B}_{w 1}^{T} \mathbf{D}_{11} \mathbf{B}_{u 1} \mathbf{q}_{u} d A+ \\
+1 / 4 \int_{A} \mathbf{q}_{u}^{T} \mathbf{B}_{u 1}^{T} \mathbf{D}_{11} \mathbf{B}_{w 1} \mathbf{W B}_{w 2} \mathbf{q}_{w} d A
\end{array}\right]+ \\
& +\left\{\begin{array}{l}
1 / 8 \int_{A} \mathbf{q}_{u}^{u T} \mathbf{B}_{u 2}^{u T} \mathbf{U}^{T} \mathbf{B}_{u 1}^{u T} \mathbf{D}_{11} \mathbf{B}_{u 1}^{u} \mathbf{U} \mathbf{B}_{u 2}^{u} \mathbf{q}_{u}^{u} d A+1 / 4 \int_{A} \mathbf{q}_{u}^{u T} \mathbf{B}_{u 4}^{u T} \mathbf{D}_{11} \mathbf{B}_{u 1}^{u} \mathbf{U} \mathbf{B}_{u 2}^{u} \mathbf{q}_{u}^{u} d A+ \\
+1 / 4 \int_{A} \mathbf{q}_{u}^{u T} \mathbf{B}_{u 2}^{u T} \mathbf{U}^{T} \mathbf{B}_{u 1}^{u T} \mathbf{D}_{11} \mathbf{B}_{u 4}^{u} \mathbf{q}_{u}^{u} d A+ \\
+1 / 4 \int_{A} \mathbf{q}_{u}^{v T} \mathbf{B}_{u 5}^{v T} \mathbf{D}_{11} \mathbf{B}_{u 1}^{u} \mathbf{U} \mathbf{B}_{u 2}^{u} \mathbf{q}_{u}^{u} d A+1 / 4 \int_{A} \mathbf{q}_{u}^{u T} \mathbf{B}_{u 2}^{u T} \mathbf{U}^{T} \mathbf{B}_{u 1}^{u T} \mathbf{D}_{11} \mathbf{B}_{u 5}^{v} \mathbf{q}_{u}^{v} d A+ \\
+1 / 8 \int_{A} \mathbf{q}_{w}^{T} \mathbf{B}_{w 2}^{T} \mathbf{W}^{T} \mathbf{B}_{w 1}^{T} \mathbf{D}_{11} \mathbf{B}_{u 1}^{u} \mathbf{U} \mathbf{B}_{u 2}^{u} \mathbf{q}_{u}^{u} d A+1 / 8 \int_{A} \mathbf{q}_{u}^{u T} \mathbf{B}_{u 2}^{u T} \mathbf{U}^{T} \mathbf{B}_{u 1}^{u T} \mathbf{D}_{11} \mathbf{B}_{w 1} \mathbf{W B}_{w 2} \mathbf{q}_{w} d A
\end{array}\right\}- \\
& -\int_{A} \mathbf{q}^{T} \mathbf{A}^{T} \mathbf{p} d A
\end{aligned}
$$

The multiplication results of the membrane and bending actions in the first bracket of Eq. (4) are uniquely defined and uncoupled, whilst those in second [von Karman assumptions] and third bracket \{Green-Lagrange approach\} are functions of the displacements $u_{0}, v_{0}$ and $w$. Consequently, the membrane and bending actions are coupled in many ways.

The conventional and the geometric stiffness matrices are, respectively: 


$$
\begin{gathered}
\left(\hat{\mathbf{K}}_{u u}=\int_{A} \mathbf{B}_{u 1}^{T} \mathbf{D}_{11} \mathbf{B}_{u 1} d A, \hat{\mathbf{K}}_{w w}=\int_{A} \mathbf{B}_{w 3}^{T} \mathbf{D}_{22} \mathbf{B}_{w 3} d A\right) \\
{\left[\tilde{\mathbf{K}}_{w w}=\int_{A} \mathbf{B}_{w 2}^{T} \mathbf{W}^{T} \mathbf{G}_{1} \mathbf{W} \mathbf{B}_{w 2} d A, \tilde{\mathbf{K}}_{w u}=\int_{A} \mathbf{B}_{w 2}^{T} \mathbf{W}^{T} \mathbf{G}_{2} d A, \tilde{\mathbf{K}}_{u w}=\int_{A} \mathbf{G}_{2}^{T} \mathbf{W} \mathbf{B}_{w 2} d A\right]} \\
\left\{\tilde{\mathbf{K}}_{u u}^{u u}=\int_{A} \mathbf{B}_{u 2}^{u T} \mathbf{U}^{T} \mathbf{G}_{3} \mathbf{U} \mathbf{B}_{u 2}^{u} d A, \tilde{\mathbf{K}}_{u u}^{u u^{*}}=\int_{A} \mathbf{G}_{4} \mathbf{U} \mathbf{B}_{u 2}^{u} d A, \tilde{\mathbf{K}}_{u u}^{u u^{* *}}=\int_{A} \mathbf{B}_{u 2}^{u T} \mathbf{U}^{T} \mathbf{G}_{4}^{T} d A,\right. \\
\tilde{\mathbf{K}}_{u u}^{v u}=\int_{A} \mathbf{G}_{5} \mathbf{U} \mathbf{B}_{u 2}^{u} d A, \tilde{\mathbf{K}}_{u u}^{u v}=\int_{A}^{u} \mathbf{B}_{u 2}^{u T} \mathbf{U}^{T} \mathbf{G}_{5}^{T} d A, \tilde{\mathbf{K}}_{w u}^{u}=\int_{A} \mathbf{B}_{w 2}^{T} \mathbf{W}^{T} \mathbf{G}_{6} \mathbf{U} \mathbf{B}_{u 2}^{u} d A, \\
\left.\tilde{\mathbf{K}}_{u w}^{u}=\int_{A}^{u} \mathbf{B}_{u 2}^{u T} \mathbf{U}^{T} \mathbf{G}_{6}^{T} \mathbf{W B}_{w 2} d A\right\} .
\end{gathered}
$$

where

$$
\begin{gathered}
{\left[\mathbf{G}_{1}=\mathbf{B}_{w 1}^{T} \mathbf{D}_{11} \mathbf{B}_{w 1}, \mathbf{G}_{2}=\mathbf{B}_{w 1}^{T} \mathbf{D}_{11} \mathbf{B}_{u 1}\right],} \\
\left\{\mathbf{G}_{3}=\mathbf{B}_{u 1}^{u T} \mathbf{D}_{11} \mathbf{B}_{u 1}^{u}, \mathbf{G}_{4}=\mathbf{B}_{u 4}^{u T} \mathbf{D}_{11} \mathbf{B}_{u 1}^{u}, \mathbf{G}_{5}=\mathbf{B}_{u 5}^{v T} \mathbf{D}_{11} \mathbf{B}_{u 1}^{u}, \mathbf{G}_{6}=\mathbf{B}_{w 1}^{T} \mathbf{D}_{11} \mathbf{B}_{u 1}^{u}\right\}
\end{gathered}
$$

The geometric stiffness matrix of structure is built by summing overlapping terms of the component strip matrices; in the same way that conventional stiffness matrix of structure is built by summing terms of the conventional strip matrices using the transformation matrices between the local and global displacements [5].

\subsection{Stability equations}

Stability equations are derived from the virtual work principle and the strain energy methods. In order to obtain the stability equations from the variational relations, the principle of the stationary potential energy will be invoked. Since the principle of the stationary potential energy states that the necessary condition of the equilibrium of any given state is that the variation of the total potential energy of the considered structure is equal to zero, we have the following relation:

$$
\delta \Pi=0 .
$$

We conclude from Eq. (7) that, if the structure is given the small virtual displacements, the equilibrium still persists if an increment of the total potential energy of the structure $\delta \Pi$ is equal to zero. Eq. (7) is the basis to derive the variational equation of equilibrium of a structure, and it is correct for both the pre- and post-critical deformation states. Eq. (7) is satisfied for an arbitrary value of the variations of parameters $\delta \mathbf{q}_{m}^{T}$. Thus we have the following conditions, which must be satisfied for any harmonic $m$ :

$$
\frac{\partial \Pi}{\partial \mathbf{q}_{m}^{T}}=\mathbf{0} .
$$

Next, we calculate derivatives of the total potential energy of a strip and finally, we get a non-homogeneous and nonlinear set of algebraic equations (9), which are the searched stability equations. 


$$
\begin{aligned}
& \left(\hat{\mathbf{K}}_{u u} \mathbf{q}_{u}+\hat{\mathbf{K}}_{w w} \mathbf{q}_{w}\right)+\left[1 / 2 \tilde{\mathbf{K}}_{w w} \mathbf{q}_{w}+1 / 2 \tilde{\mathbf{K}}_{w u} \mathbf{q}_{u}+1 / 4 \tilde{\mathbf{K}}_{u w} \mathbf{q}_{w}\right]+ \\
+ & \left\{\begin{array}{l}
1 / 2 \tilde{\mathbf{K}}_{u u}^{u u} \mathbf{q}_{u}^{u}+3 / 4 \tilde{\mathbf{K}}_{u u}^{u u^{*}} \mathbf{q}_{u}^{u}+3 / 4 \tilde{\mathbf{K}}_{u u}^{u u^{* *}} \mathbf{q}_{u}^{u}+1 / 4 \tilde{\mathbf{K}}_{u u}^{v u} \mathbf{q}_{u}^{u}+ \\
+1 / 2 \tilde{\mathbf{K}}_{u u}^{u v} \mathbf{q}_{u}^{v}+1 / 4 \tilde{\mathbf{K}}_{w u}^{u} \mathbf{q}_{u}^{u}+1 / 4 \tilde{\mathbf{K}}_{u w}^{u} \mathbf{q}_{w}
\end{array}\right\}-\mathbf{Q}=\mathbf{0}
\end{aligned}
$$

We can visualize the construction of a strip stiffness matrix, which is composed of twelve block matrices. Assembling block matrices into conventional/geometric stiffness matrix of each strip is performed according to the scheme presented in Fig. 2, where: $\mathrm{ST} 1=\hat{\mathbf{K}}_{u u}, \mathrm{ST} 2=\hat{\mathbf{K}}_{w w}, \mathrm{ST} 3=\tilde{\mathbf{K}}_{w w}, \mathrm{ST} 4=\tilde{\mathbf{K}}_{w u}, \mathrm{ST} 5=\tilde{\mathbf{K}}_{u w}, \mathrm{ST} 6=\tilde{\mathbf{K}}_{u u}^{u u}, \mathrm{ST7}=\tilde{\mathbf{K}}_{u u}^{u u^{*}}, \mathrm{ST} 8=\tilde{\mathbf{K}}_{u u}^{u *^{* *}}$, $\mathrm{ST} 9=\tilde{\mathbf{K}}_{u u}^{v u}, \mathrm{ST} 10=\tilde{\mathbf{K}}_{u u}^{u v}, \mathrm{ST} 11=\tilde{\mathbf{K}}_{w u}^{u}$ and ST12 $=\tilde{\mathbf{K}}_{u w}^{u}\left(\mathrm{ST} 5=\mathrm{ST} 4^{\mathrm{T}}, \mathrm{ST} 8=\mathrm{ST}^{\mathrm{T}}, \mathrm{ST} 10=\mathrm{ST} 9^{\mathrm{T}}\right.$, $\mathrm{ST} 12=\mathrm{ST} 11^{\mathrm{T}}$ ).

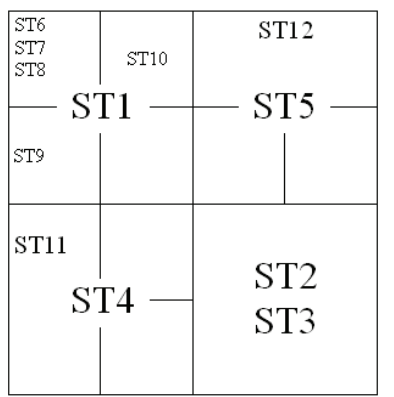

Fig. 2. Strip stiffness matrix assembling

\subsection{Solution of nonlinear equations}

For equilibrium, the principle of stationary potential energy requires that:

$$
\mathbf{R}=\partial \Pi / \partial \mathbf{q}^{T}=[\hat{\mathbf{K}}+\tilde{\mathbf{K}}] \mathbf{q}-\mathbf{Q}=\mathbf{K} \mathbf{q}-\mathbf{Q}=\mathbf{0}
$$

where $\Pi$ is a function of the displacements $\boldsymbol{q}$, and $\boldsymbol{R}$ represent the gradient or residual force vector, which is generally nonzero for some approximate displacement vector $\boldsymbol{q}_{0}$ (the subscript 0 denotes an old value). It is assumed that a better approximation is given by:

$$
\mathbf{q}_{n}=\mathbf{q}_{0}+\boldsymbol{\delta}_{0} .
$$

where subscript $n$ denotes a new value.

Taylor's expansion of Eq. (10) yields:

$$
\mathbf{R}_{n}=\mathbf{R}\left(\mathbf{q}_{0}+\boldsymbol{\delta}_{0}\right)=\mathbf{R}\left(\mathbf{q}_{0}\right)+\overline{\mathbf{K}}_{0} \boldsymbol{\delta}_{0}+\ldots=\mathbf{R}_{0}+\overline{\mathbf{K}}_{0} \boldsymbol{\delta}_{0}+\ldots
$$

where $\overline{\mathbf{K}}_{0}=\partial \mathbf{R} / \partial \mathbf{q}$ is the matrix of second partial derivatives of $\Pi$ calculated at $\boldsymbol{q}_{0}$ (i.e. the tangent stiffness matrix or Hessian matrix). Setting Eq. (12) to zero and considering only linear terms in $\boldsymbol{\delta}_{\boldsymbol{0}}$ gives the standard expression for Newton-Raphson iteration:

$$
\boldsymbol{\delta}_{0}=-\overline{\mathbf{K}}_{0}^{-1} \mathbf{R}_{0} .
$$


Using this approach, a further iteration yields:

$$
\boldsymbol{\delta}_{n}=\overline{\mathbf{K}}_{n}^{-1} \mathbf{R}_{n}
$$

where $\overline{\mathbf{K}}_{n}=\partial \mathbf{R} / \partial \mathbf{q}$ at $\boldsymbol{q}_{n}$.

In addition, the blocks of the conventional and geometric tangent stiffness matrix of each strip are:

$$
\begin{gathered}
\hat{\overline{\mathbf{K}}}=\left[\begin{array}{cc}
\hat{\mathbf{K}}_{u u} & \mathbf{0} \\
\mathbf{0} & \hat{\mathbf{K}}_{w w}
\end{array}\right] . \\
\tilde{\overline{\mathbf{K}}}=\left[\begin{array}{cc}
\mathbf{0} & 1 / 2 \tilde{\mathbf{K}}_{u w} \\
1 / 2 \tilde{\mathbf{K}}_{w u} & 3 / 2 \tilde{\mathbf{K}}_{w w}
\end{array}\right]+ \\
+\left[\begin{array}{cc}
3 / 2 \tilde{\mathbf{K}}_{u u}^{u u}+3 / 2 \tilde{\mathbf{K}}_{u u}^{u u^{*}}+3 / 2 \tilde{\mathbf{K}}_{u u}^{u u^{* *}} & 1 / 2 \tilde{\mathbf{K}}_{u u}^{u v} \\
1 / 2 \tilde{\mathbf{K}}_{u u}^{v u} & \mathbf{0}
\end{array}\right]+\left[\begin{array}{cc}
\mathbf{0} & 1 / 2 \tilde{\mathbf{K}}_{u w}^{u} \\
1 / 2 \tilde{\mathbf{K}}_{w u}^{u} & \mathbf{0}
\end{array}\right] .
\end{gathered}
$$

Comparing these expressions with Eq. (9), it is apparent that the conventional stiffness matrix remains unchanged, while the geometric tangent matrix becomes symmetrical.

\subsection{Static buckling}

The loss of stability of static equilibrium states of structures subjected to conservative loads is in general known as static buckling of the structure. For conservative systems, the principle of minimum of the total potential energy can be used to test the stability of a structure (static equilibriums are extremes of the total potential energy). The Hessian with respect to the local DOFs is denoted as the tangent stiffness matrix, of each strip i.e.:

$$
\overline{\mathbf{K}}=[\hat{\overline{\mathbf{K}}}+\tilde{\overline{\mathbf{K}}}] .
$$

The (local) stability of equilibrium states of conservative systems by HCFSM can be assessed by looking at the eigenvalues of the tangent stiffness matrix of structure $\overline{\mathbf{K}}_{(D O F s \cdot r \cdot(n+1)) \cdot(D O F s \cdot r \cdot(n+1))}$ with $(n+1)$ nodal lines (n=number of strips), which are all real, since tangent stiffness matrix of the strip is a symmetric matrix.

Let $\lambda_{i}$ denote the $i^{\text {th }}$ eigenvalue of the tangent stiffness matrix of structure. Based on theorems of Lagrange-Dirichlet and Lyapunov [8] it can be concluded that an equilibrium state is stable if all $\lambda_{i}>0$, while an equilibrium state is unstable if one or more $\lambda_{i}<0$. If along a load-path, at some equilibrium state one or more $\lambda_{i}=0$, this equilibrium state is denoted as a critical state. Static buckling refers in general to case where, starting from some stable state, a critical state is reached along the load-path.

In general, static buckling is solved by solving Eq. (9) for a varying load $P$ with, for example, some sort of numerical path-following routine [5], while simultaneously tracking the eigenvalues of the tangent stiffness matrix of structure. Buckling occurs where the matrix becomes singular. 


\section{Application to Prismatic Folded Plate StRUCtures}

\subsection{Subject and method of analysis}

Prismatic folded plates are spatial carrying structures composed of thin flat rectangular plates monolithically connected at a certain angle along the longitudinal edges (see Fig. 1a). Historical overview of methods for analysis of folded plate structure (FPS) is given in [9]. The choice of adequate method largely depends on the geometrical characteristics of the structure. The length/width ratio of the structure as a whole, and of individual plates, affects the possibility to neglect the impact of certain action effects in equations of equilibrium, which simplifies the process of analysis, without significant diminishing the accuracy of the results. The assumption of small displacements of the "short" FPS is often justified, while the calculation of "long" one should be carried out taking into account the large deflections. All this highlights the need to determine more clearly the boundary between "short" and "long" folded plate structures. This must be performed using the method of analysis whose applicability does not depend on the length/width ratio, such as numerical methods - FEM and FSM.

Bearing in mind that in FSM appears considerably smaller number of equations, matrices and input-output data than in FEM, which makes this method less demanding in terms of capacity and computer time required for execution, and often easier for its application, for the analysis of this special class structures in [9] is recommended the use of FSM.

The shape of cross-section of reinforced concrete prismatic FPS is determined according to technological, structural, architectural and other requirements. Optimal dimensions and slope of individual elements of such formed structure should be determined by analyzing several alternative solutions. The first step in this analysis is to determine and compare the diagrams of displacements, rotations, internal forces and stresses of various cross sections.

Reinforced concrete simple supported prismatic folded plate structures, with the total width of $B=11.6 \mathrm{~m}$, are analyzed (Figs. $4,13,14$ and 15). Vertical uniformly distributed load: self-weight of structure $g$, the weight of covering on sloped plates $\Delta \mathrm{g}=0.5 \mathrm{kN} / \mathrm{m}^{2}$ and snow load $\mathrm{s}=1.0 \mathrm{kN} / \mathrm{m}^{2}$, is applied. Structures are made of concrete MB $45(\mathrm{C} 35 / 45)$ with reinforcement RA 400/500-2 (B400).

Structural analysis is performed by FSM usig one hundred series terms. The symmetry of geometry, loads and supporting conditions is utilized, so the numerical analysis is carried out for only half of the structure. The finite strip mesh (of 10 strips), their labels and labels of nodal lines, as well as the orientation of the global coordinate system, are shown in Figs. 4, 13, 14 and 15. Each strip has the following elastic material properties: elastic modulus E $=34 \mathrm{GPa}$ and Poisson's ratio $v=0$.

\subsection{Determining the boundary between "short" and "long" FPS}

To determine the boundary between "short" and "long" FPSs, reinforced concrete prismatic folded plate structure of cross-section shown in Fig 4 is analyzed. The length of structure is varied from $\mathrm{L}=5 \mathrm{~m}$, with corresponding length/width ratio of individual plates $1_{\mathrm{y}} / 1_{\mathrm{x}}=1.59$, to $\mathrm{L}=100 \mathrm{~m}\left(\mathrm{l}_{\mathrm{y}} / \mathrm{l}_{\mathrm{x}}=31.87\right)$. 


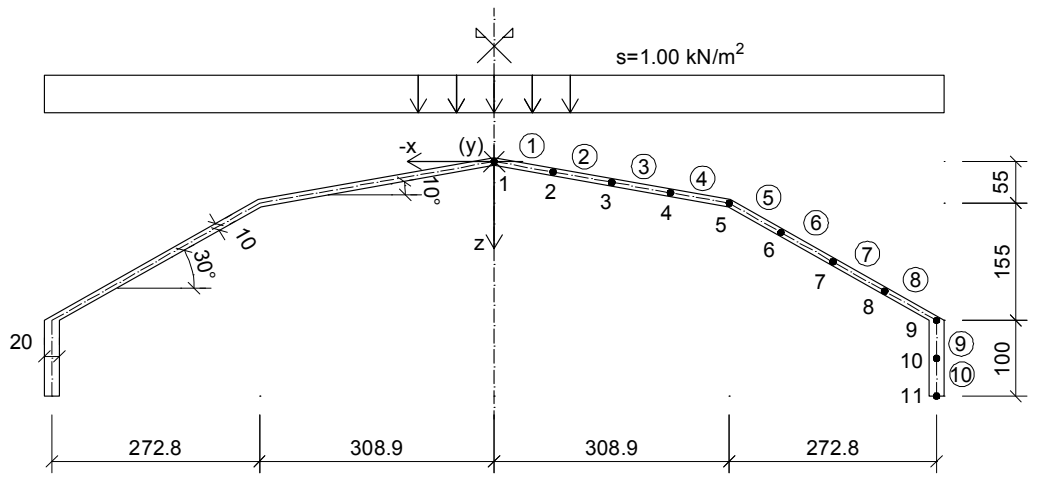

Fig. 4. Cross-section and load of the FPS type "A"
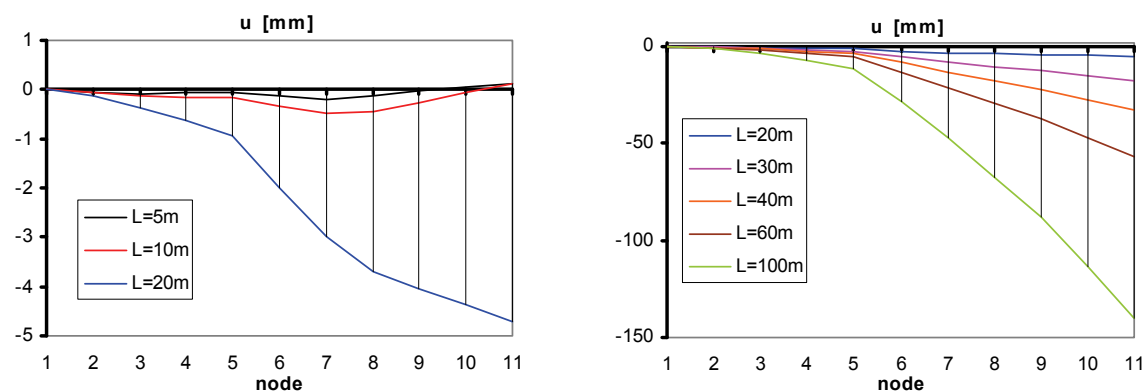

Fig. 5. Displacements in direction of the global " $\mathrm{x}$ " axis at midspan

Observing distribution of displacements, rotations, internal forces, moments and stresses (Figs. 5 to 11), due to the total service load, for various span lengths of FPSs, some notices can be made.
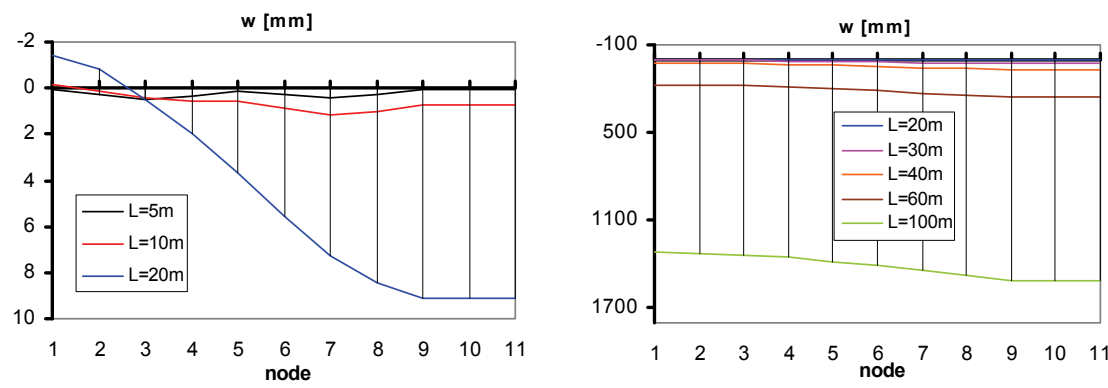

Fig. 6. Vertical deflection at midspan 

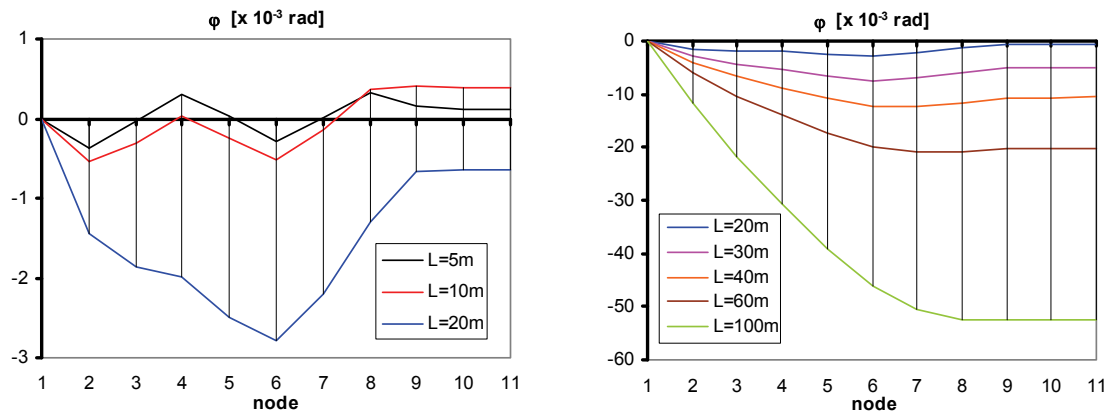

Fig. 7. Rotation angles about the longitudinal axis at midspan

Bottom line of edge beams (node line 11), of spans $\mathrm{L}=5 \mathrm{~m}$ and $\mathrm{L}=10 \mathrm{~m}$, moves outward (Fig. 5), while in all other spans it moves inward (toward the axis of symmetry of the cross section). Ending with $\mathrm{L}=20 \mathrm{~m}$ distribution of horizontal displacements between nodal lines 5 and 9 is parabolic, while for longer spans it is almost linear.

In spans $\mathrm{L}=10 \mathrm{~m}$ and $\mathrm{L}=20 \mathrm{~m}$, points near the axis of symmetry of the cross section are moving upward (negative displacements). In all other span lengths, all points of the cross section have positive vertical deflection (Fig. 6).

The angle of rotation about the longitudinal axis of the structure (Fig. 7) has variable sign along the cross section only at span lengths $\mathrm{L}=5 \mathrm{~m}$ and $\mathrm{L}=10 \mathrm{~m}$.

The distribution of longitudinal force $\mathrm{N}_{\mathrm{y}}$ of long structures is almost linear along the width of plates (Fig. 8).
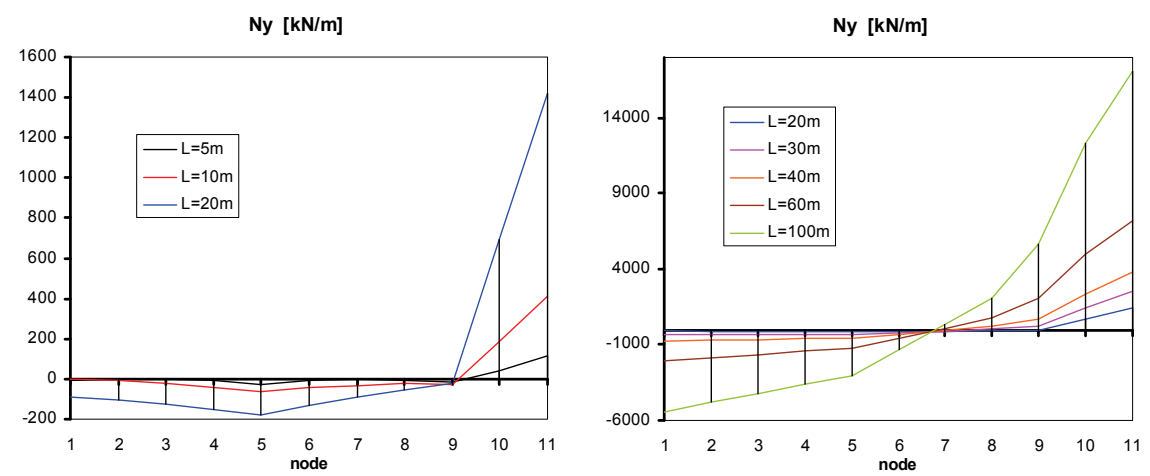

Fig. 8. Longitudinal force $\mathrm{N}_{\mathrm{y}}$ at midspan

For span lengths up to $\mathrm{L}=20 \mathrm{~m}$ the maximum value of longitudinal stress in pressure is not reached at the highest point of cross section (nodal line 1), but slightly below it (Fig. 9). Increasing span length reduces the height of pressured area. For very large spans $(\mathrm{L}=100 \mathrm{~m})$ distribution of longitudinal stresses becomes linear. 

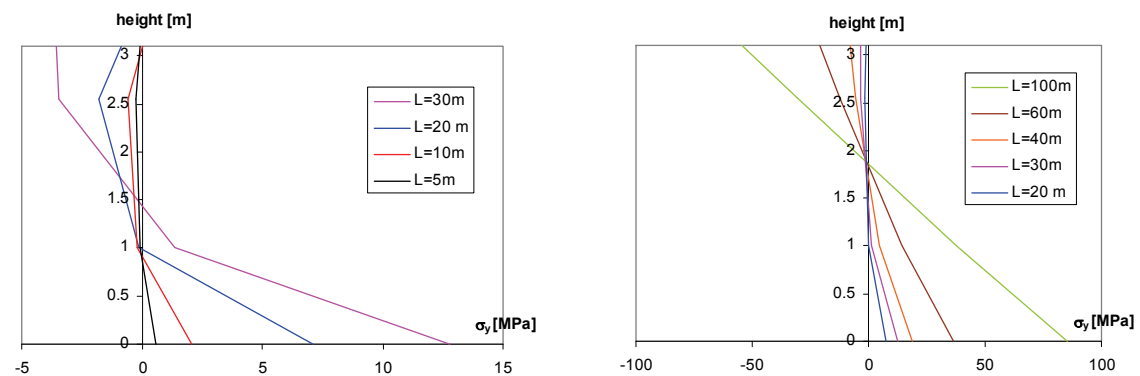

Fig. 9. Longitudinal stress $\sigma_{\mathrm{y}}$ at midspan, along the height of cross section
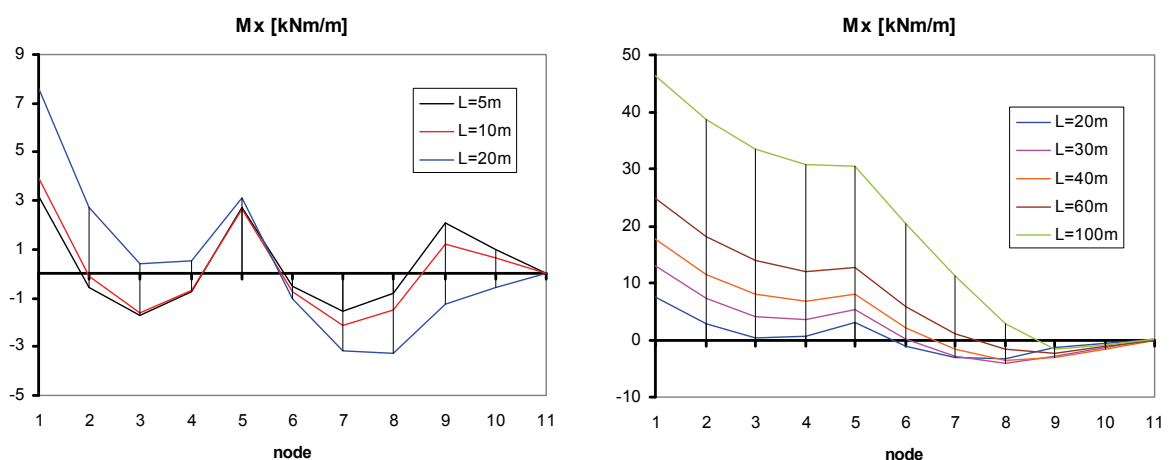

Fig. 10. Transverse moment $M_{x}$ at midspan
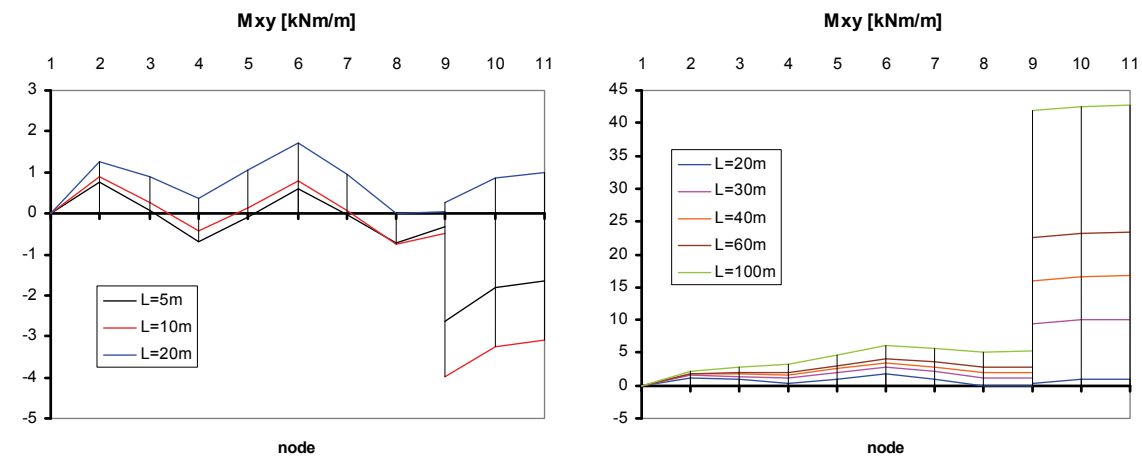

Fig. 11. Torsional moment $\mathrm{M}_{\mathrm{xy}}$ at support

According to these findings, two different behaviors can be observed: for structures of length up to $\mathrm{L}=10 \mathrm{~m}$, with corresponding length/width ratio of individual plates of $1_{\mathrm{y}} / 1_{\mathrm{x}}=3.19$, which may be considered as "short", and for "long" ones, of span longer than $\mathrm{L}=20 \mathrm{~m}\left(\mathrm{l}_{\mathrm{y}} / \mathrm{l}_{\mathrm{x}}=6.37\right)$. More precise border may be obtained only comparing the results of FSM and HCFSM analysis. 
Using the von Karman and the Green-Lagrange HCFSM solutions, the large-displacement stability analysis of reinforced concrete FPS, shown in Fig. 4, is performed for span lengths of $10,15,20,25$ and $30 \mathrm{~m}$. 31 series terms are adopted in computations. The total loading is divided into 8 load increments. The load factor 0.8 corresponds to the service load. Fig. 12 shows that the effect of nonlinear behavior is not much pronounced in $20 \mathrm{~m}$ long structure.
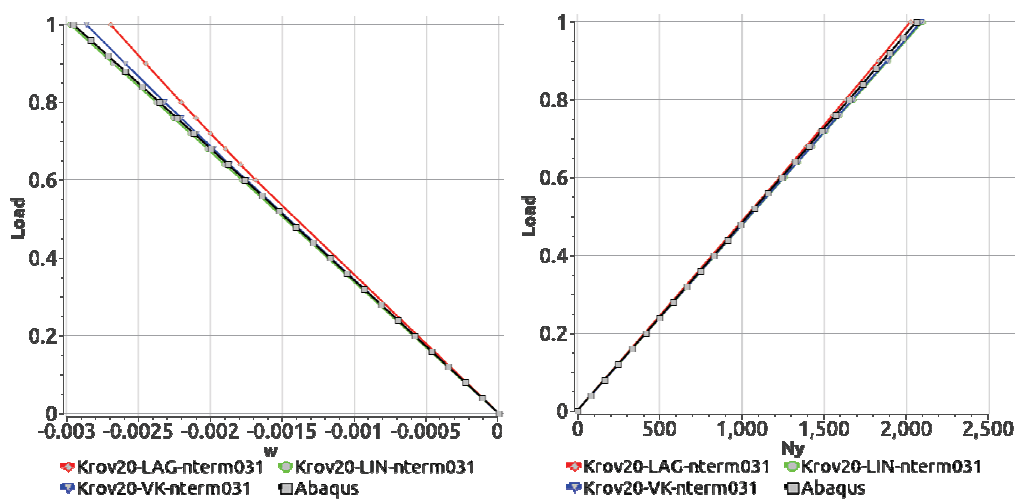

Fig. 12. Variation of central deflection $w$ in nodal line 1 and longitudinal force $N_{y}$ in nodal line 11 with load intensity, using HCFSM

The comparative analysis of HCFSM and FSM results shows that the assumption of small displacements is fully justified for span lengths up to $15 \mathrm{~m}\left(\mathrm{~L} / \mathrm{B}=1.29,1_{\mathrm{y}} / 1_{\mathrm{x}}=4.8\right)$. Geometric nonlinear effects need to be taken into consideration for longer structures, in order to obtain realistic numerical solutions.

\subsection{Selection of the optimal cross-section of FPS}

Reinforced concrete simple supported $20 \mathrm{~m}$ long folded plate structures of four different cross-sections, type A to D (Figs. 4, 13, 14 and 15), are analyzed.

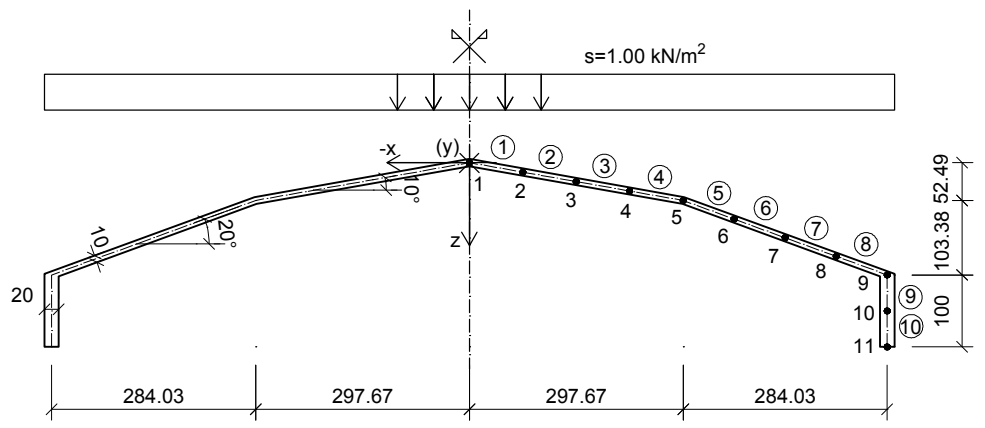

Fig. 13. Cross-section and load of the FPS type "B" 


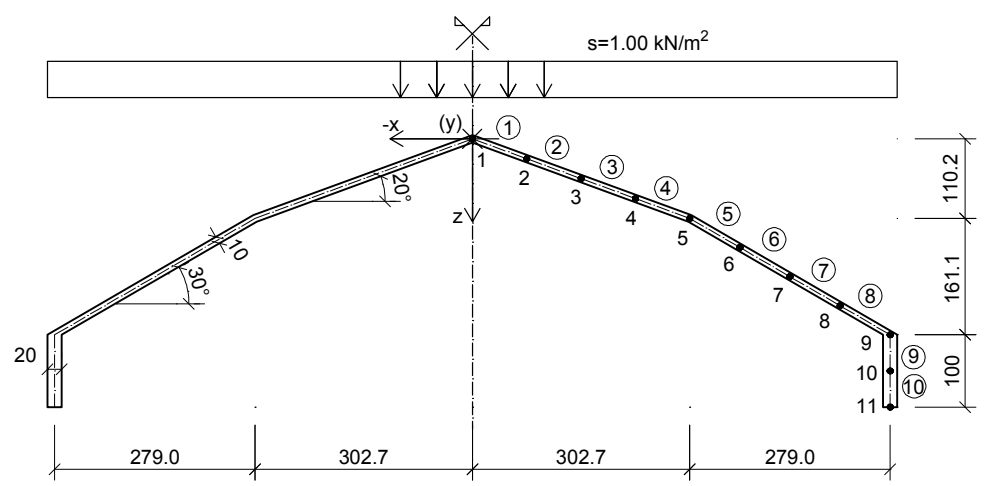

Fig. 14. Cross-section and load of the FPS type "C"

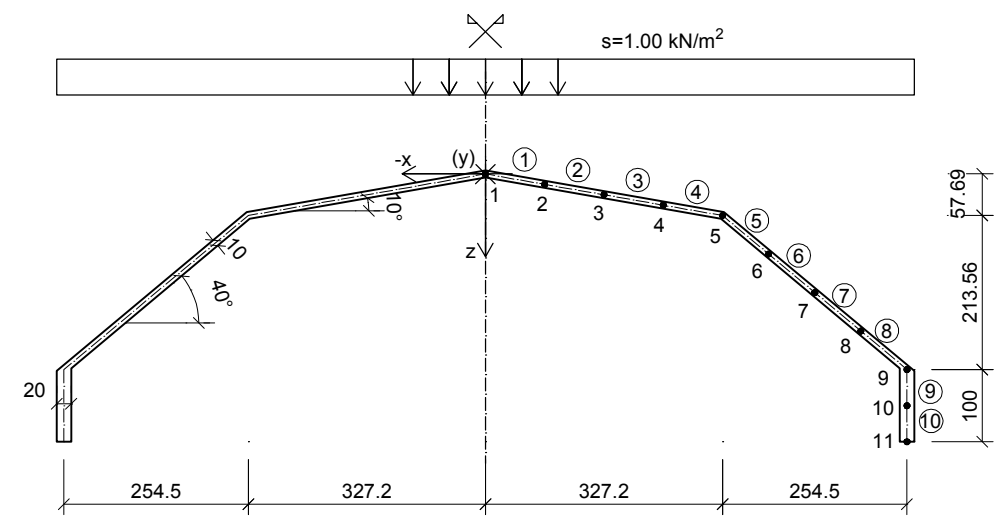

Fig. 15. Cross-section and load of the FPS type "D"

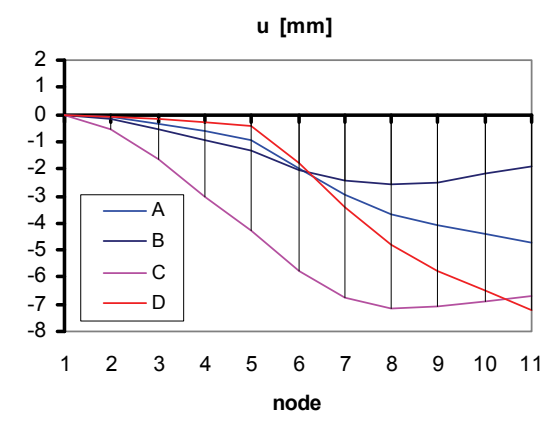

Fig. 16. Displacements in direction of the global " $\mathrm{x}$ " axis at midspan

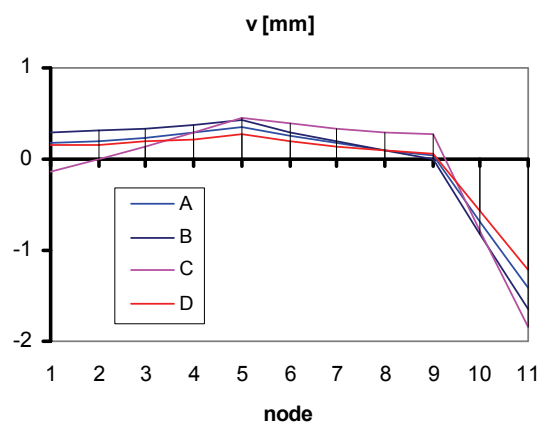

Fig. 17. Displacements in direction of the global "y" axis at supports 


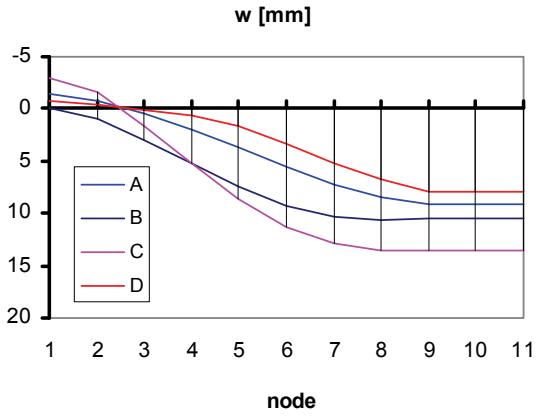

Fig. 18. Vertical deflections at midspan

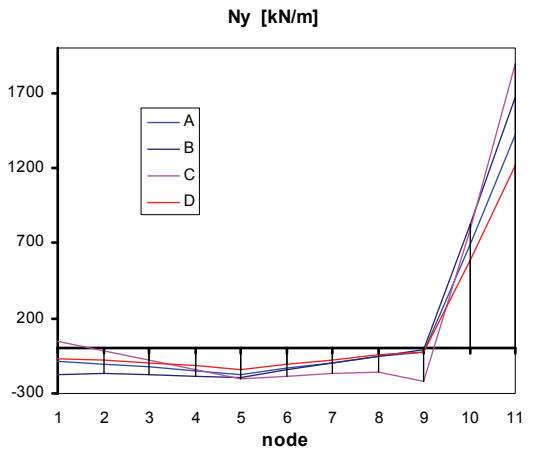

Fig. 20. Longitudinal force $\mathrm{N}_{\mathrm{y}}$ at midspan

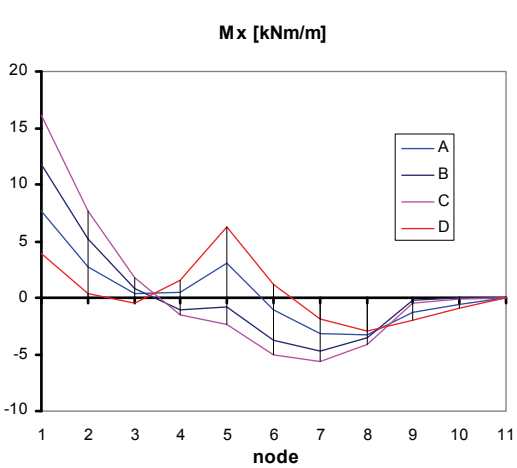

Fig. 22. Transverse moment $M_{x}$ at midspan

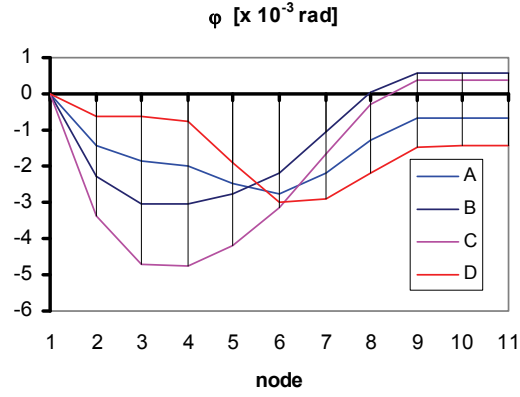

Fig. 19. Rotation angles about the longitudinal axis at midspan

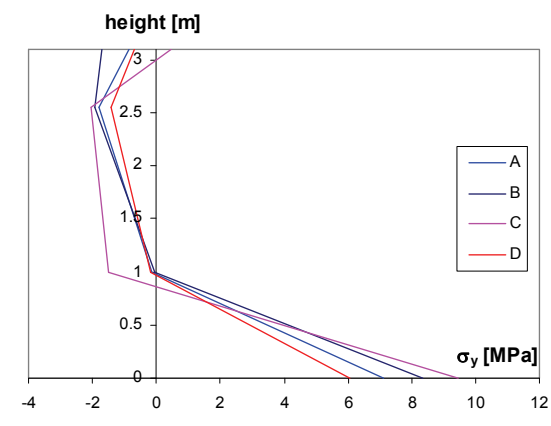

Fig. 21. Longitudinal stress $\sigma_{\mathrm{y}}$ at midspan, along the height of cross section

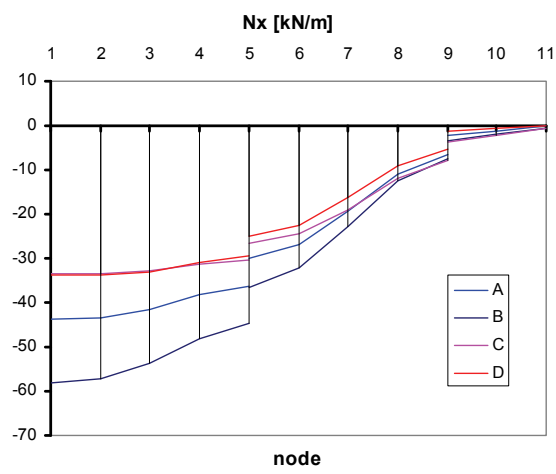

Fig. 23. Transverse membrane force $N_{x}$ at midspan 


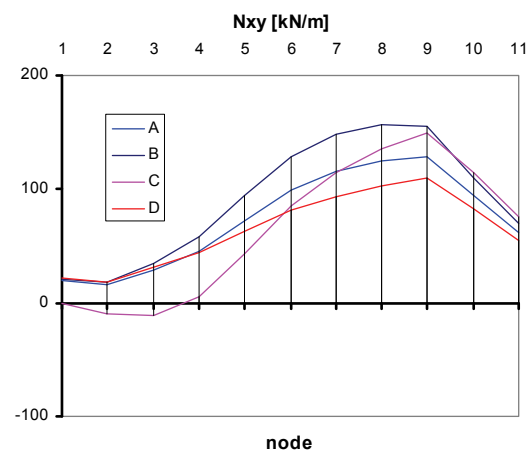

Fig. 24. Membrane shear $\mathrm{N}_{\mathrm{xy}}$ at support

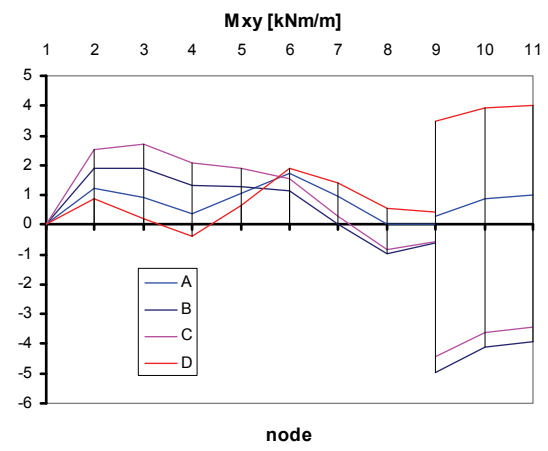

Fig. 25. Torsional moment $\mathrm{M}_{\mathrm{xy}}$ at support

Based on comparative analysis of displacements, rotations, internal forces, stresses and moments (Figs. 16 to 25), due to the total service load, obtained for different types of the cross section, it can be concluded that cross section of the FPS type "C" (Fig. 14) adverse, and it should be avoided. Due to large shear forces and torsional moments, which occur in sloped plates, too "shallow" cross sections (type "B") of reinforced concrete FPS should be avoided, too. Best behavior showed FPSs type "A" and "D". How torsional moments in reinforced concrete edge beam may be crucial for its dimensioning, it is recommended to use cross section of type "A", in which these effects are the smallest in comparison to all other analyzed types of cross section.

\section{CONCLUSINS}

The problem of finding the optimal shape, size and topology of reinforced prismatic folded plate structures under multiple loading conditions has been investigated. The structural analysis needed for the optimal design process have been performed by using the FSM and HCFSM methods. A number of applications have shown the effectiveness of the proposed procedure. Clearly, future developments are expected in order to obtain a better control of the design problem, especially on the definition of the design constraints, which should also account for technological aspects, as well as for aesthetical and additional functional requirements.

\section{REFERENCES}

1. ASCE, Design of Cylindrical Concrete Shell Roofs, Manual of Engineering Practice, New York (NY): ASCE, 1952, p.31

2. V. Kristek, Theory of Box Girder Bridges, Prague, John Wiley and Sons, 1979.

3. Y. C. Loo and A. Cusens, The Finite Strip Method in Bridge Engineering, London, E. \& F.N, Spon, 1978.

4. Y. K. Cheung and L. G, Tham. Finite Strip Method, Boca Raton, CRC Press, 1998.

5. D. D. Milašinović, The Finite Strip Method in Computational Mechanics, Faculties of Civil Engineering, University of Novi Sad, Technical University of Budapest and University of Belgrade, Subotica, Budapest, Belgrade, 1997. 
6. D. D. Milašinović,"Geometric non-linear analysis of thin plate structures using the harmonic coupled finite strip method", Thin-Walled Structures, 49(2), pp. 280-290, 2011.

7. J. M. Davies, "Generalized beam theory (GBT) for coupled instability problems", in J. Rondal (Ed.), Coupled Instabilities in Metal Structures, Theoretical and Design Aspects, CISM Courses and Lectures N. 379, p. 151-223, Wien-NY, Springer-Verlag, 2000.

8. Z. P. Bažant and L. Cedolin, Stability of Structures: Elastic, Inelastic, Fracture, and Damage Theories, Oxford Univeristy Press, Oxford, 1991.

9. D. Goleš, Rheological-Dynamical Analysis of Reinforced Concrete Folded Plates, PhD thesis, Faculty of Civil Engineering, University of Novi Sad, 2012.

\section{OPTIMALNO PROJEKTOVANJE ARMIRANOBETONSKIH POLIEDARSKIH LJUSKI MODELIRANJEM METODOM KONAČNIH TRAKA}

\section{Dragan D. Milašinović, Danica Goleš}

Prikazano je optimalno projektovanje armiranobetonskih poliedarskih ljuski za složene uslove opterećenja. Projektne promenjive uključuju geometrijske veličine kao što su debljine, dimenzije strukturalnih elemenata (ploča) $i$ topološke parametre koji definišu položaj $i$ povezanost elemenata. Proračunska analiza je izvršena primenom klasičnog i kompleksnog harmonijski spojenog metoda konačnih traka za rešavanje odgovarajućeg geometrijski nelinearnog problema. Efikasnost predloženih procedura je pokazana na primeru optimalnog projektovanja prizmatičnih poliedarskih ljuski.

Ključne reči: metod konačnih traka, poliedarske ljuske, armirani beton. 